HUB EP-98/28

hep-th/9805110

\title{
Duality of Self-Dual Actions
}

\author{
Alexey Maznytsia ${ }^{1}$, Christian R. Preitschopf ${ }^{2}$ and Dmitri Sorokin ${ }^{2}{ }^{3}$ 冈 \\ ${ }^{1}$ Department of Physics and Technology, Kharkov State University \\ 310108, Kharkov, Ukraine \\ e-mail: alex_maznytsia@hotmail.com \\ ${ }^{2}$ Humboldt-Universität zu Berlin \\ Institut für Physik \\ Invalidenstrasse 110, D-10115 Berlin, Germany \\ e-mails: preitsch,sorokin@physik.hu-berlin.de \\ ${ }^{3}$ Università Degli Studi Di Padova \\ Dipartimento Di Fisica "Galileo Galilei" \\ ed INFN, Sezione Di Padova \\ Via F. Marzolo, 8, 35131 Padova, Italia
}

\begin{abstract}
Using examples of a $D=2$ chiral scalar and a duality-symmetric formulation of $D=4$ Maxwell theory we study duality properties of actions for describing chiral bosons. In particular, in the $D=4$ case, upon performing a duality transform of an auxiliary scalar field, which ensures Lorentz covariance of the action, we arrive at a new covariant dualitysymmetric Maxwell action, which contains a two-form potential as an auxiliary field. When the two-form field is gauge fixed this action reduces to a duality-symmetric action for Maxwell theory constructed by Zwanziger. We consider properties of this new covariant action and discuss its coupling to external dyonic sources. We also demonstrate that the formulations considered are self-dual with respect to a dualization of the field-strengths of the chiral fields.
\end{abstract}

PACS numbers: 11.15-q, 11.17+y

Keywords: Chiral bosons, Maxwell theory, electric-magnetic duality

*Alexander von Humboldt Fellow

On leave from Kharkov Institute of Physics and Technology, Kharkov, 310108, Ukraine 


\section{Introduction}

Chiral bosons, or antisymmetric tensor fields with self-dual field strengths, appear in various theoretical models related to superstring theories, and reflect the existence of a variety of important dualities connecting these theories amongst each other.

Chiral field potentials are differential $\mathrm{p}$-forms and, hence, exist in a space whose dimension is $D=2(p+1)$. If the metric signature of this space is Lorentzian, then (anti-)self-duality requires the potential to be real, if $p$ is even, or complex, if $p$ is odd. In the latter case the theory can also be described by two real antisymmetric tensor fields related by a duality condition.

The problem of a Lagrangian description of chiral bosons turned out to be a complicated one, since manifest duality and space-time covariance do not like to live in harmony with each other in one action. Several non-covariant [1]-[7] and space-time covariant [8][10] approaches have been developed to solve this problem.

The first non-covariant duality-symmetric action was proposed by Zwanziger for the description of $D=4$ Maxwell fields interacting with electrically and magnetically charged particles [1]. In this formulation an electromagnetic field is described by an $O(2)$ doublet of vector potentials $A_{m}^{\alpha}, \alpha=1,2$, whose stress tensors $F_{m n}^{\alpha}(A)=2 \partial_{[m} A_{n]}^{\alpha} \equiv \partial_{m} A_{n}^{\alpha}-\partial_{n} A_{m}^{\alpha}$ satisfy, in the absence of charged sources, the duality condition

$$
\mathcal{F}_{m n}^{\alpha}(A) \equiv \epsilon^{\alpha \beta} F_{m n}^{\beta}(A)-\frac{1}{2} \epsilon_{m n p q} F^{\alpha p q}(A)=0 ; \quad \mathcal{F}_{m n}^{\alpha}(A)=\frac{1}{2} \epsilon^{\alpha \beta} \epsilon_{m n p q} \mathcal{F}^{\beta p q}(A),
$$

where $\epsilon^{\alpha \beta}$ is the antisymmetric tensor $\left(\epsilon^{12}=1\right)$.

This condition follows from the Zwanziger action without charged sources

$$
S_{Z}=\int d^{4} x\left[-\frac{1}{8} F_{m n}^{\alpha}(A) F^{\alpha m n}(A)+\frac{1}{4} n^{m} \mathcal{F}_{m n}^{\alpha}(A) \mathcal{F}^{\alpha n p}(A) n_{p}\right],
$$

which contains a constant normalized space-like vector $n^{m}\left(n^{m} n_{m}=1\right)$ associated with a rigid Dirac string [四. Its presence breaks the $S O(1,3)$ Lorentz invariance of the action (2) down to an $S O(1,2)$ group of rotations in the hypersurface orthogonal to $n^{m}$. Nevertheless, as we will show in this paper, the action (2) is invariant under non-manifest modified space-time transformations which coincide with the $S O(1,3)$ Lorentz transformations on the mass shell.

To reduce the $A_{m}^{\alpha}$-field equations of motion derived from (2) to the duality condition (II) one should impose appropriate boundary conditions on the field strengths of the gauge fields [1].

Another non-covariant duality-symmetric action for Maxwell theory was considered by Deser and Teitelboim [2], and by Schwarz and Sen [7]. This action can be written in the following form:

$$
S=\int d^{4} x\left[-\frac{1}{8} F_{m n}^{\alpha}(A) F^{\alpha m n}(A)-\frac{1}{4} n^{m} \mathcal{F}_{m n}^{\alpha}(A) \mathcal{F}^{\alpha n p}(A) n_{p}\right] .
$$

It also contains a normalized constant space-like vector $n^{m}$. We observe that the two actions differ by a sign in front of the second term. However, they both produce the

\footnotetext{
${ }^{1}$ In [2, 7] a time-like vector $n^{m}=(1,0,0,0)$ was used to construct the action, but the choice of a constant space-like vector is equally admissible.
} 
duality condition (11) and describe a single Maxwell field. This sign difference results in a difference in symmetry properties of the actions. For instance, the duality condition arises as a consequence of equations of motion, which follow from the action (3), upon gauge fixing an additional local symmetry

$$
\delta A_{m}^{\alpha}=n_{m} \Phi^{\alpha}(x)
$$

under which this action is invariant. The Zwanziger action does not possess such a symmetry.

A purpose of this paper is to establish the relationship between these two actions. We will show that the Schwarz-Sen action and the Zwanziger action are dual to each other. To perform the duality transform from one action to another one should consider a manifestly covariant form of the action (3) proposed in [10], which contains an auxiliary scalar field $a(x)$ responsible for Lorentz covariance.

We analyze properties of the covariant formulation with respect to the dualization of chiral boson fields and of the auxiliary scalar field and find that the Zwanziger action appears upon the dualization of the auxiliary field. In this way we get a new covariant form of the duality-symmetric action from which the Zwanziger action is obtained after gauge fixing a local symmetry. We also find a non-manifest space-time invariance of the action (2).

The paper is organized as follows.

As an instructive simple example, in Section 2 we consider a chiral scalar in twodimensional space-time. We demonstrate that its covariant action is self-dual with respect to a dualization of the chiral scalar as well as of the auxiliary scalar, and show that in this case the actions of the Floreanini and Jackiw [4] (or of a type (3)) and of the Zwanziger type coincide.

In Section 3 we consider the same dualization procedure in application to the dualitysymmetric formulation of $D=4$ Maxwell theory. We find that the dualization of the auxiliary scalar field $a(x)$ of this formulation into a two-rank tensor field $B_{m n}(x)$ results in another duality-symmetric action. We study symmetry structure and dynamical equations of this new covariant action and show that it reduces to the Zwanziger action (2) by gauge fixing its local symmetries.

In the Conclusion we discuss a problem of the duality relation between the actions (2) and (3) when they are coupled to electrically and magnetically charged sources.

In the Appendix we demonstrate that chiral boson actions are self-dual with respect to a dualization of chiral boson field strengths.

\section{Duality properties of the $D=2$ chiral boson action}

We begin with the consideration of a covariant action for a two-dimensional chiral scalar 11, 12

$$
S=\int d^{2} x\left[-\frac{1}{2} F_{m}(\phi) F^{m}(\phi)+\frac{1}{2\left(\partial_{r} a\right)\left(\partial^{r} a\right)}\left(\partial^{m} a \mathcal{F}_{m}(\phi)\right)^{2}\right],
$$

where $F_{m}(\phi)=\partial_{m} \phi$ is the "field strength" of the scalar field $\phi(x)$, and $\mathcal{F}_{m}(\phi)=F_{m}(\phi)-$ $\epsilon_{m n} F^{n}(\phi)$ is its anti-self-dual part

$$
\mathcal{F}_{m}(\phi)=-\epsilon_{m n} \mathcal{F}^{n}(\phi)
$$


To be space-time covariant the action (5) contains a normalized derivative of an auxiliary scalar field $a(x)$.

The action (5) is invariant under the following transformations of the fields $a(x)$ and $\phi(x)$ [11, 12, 13]

$$
\begin{gathered}
\delta a=\varphi(x), \quad \delta \phi=\frac{\varphi}{(\partial a)^{2}} \mathcal{F}^{m}(\phi) \partial_{m} a, \\
\delta \phi=f(a),
\end{gathered}
$$

with the parameters $\varphi(x)$ and $f(a)$, respectively. Note that the latter is a function of $a(x)$. Therefore, the transformations (7) are only "semi-local", and there is no firstclass constraint associated with this symmetry in the covariant formulation. The only first-class constraint is the one which generates the transformations (6] [12].

The symmetry (6) allows one to gauge away the auxiliary scalar field $a(x)$ by fixing, for example, the temporal gauge $\partial_{m} a=\delta_{m}^{0}$. Then the action (5) reduces to the noncovariant action of Floreanini and Jackiw 四, which is invariant under (7) with $f\left(x^{0}\right)$. Now there appears a 'weak' first-class constraint associated with this constraint but it rather corresponds to choosing boundary conditions for $\phi(x)$ than eliminating a physical degree of freedom of the chiral boson (see, for example [14]).

The $\phi(x)$ field equation, following from (5), is

$$
\epsilon^{m n} \partial_{n}\left[\frac{1}{2(\partial a)^{2}} \partial_{m} a \partial_{p} a \mathcal{F}^{p}(\phi)\right]=0 .
$$

Its general solution

$$
\frac{1}{2(\partial a)^{2}} \partial_{m} a \partial_{p} a \mathcal{F}^{p}(\phi)=\partial_{m} \zeta
$$

contains a scalar field $\zeta(x)$, which is supposed to be a continuous and differentiable function of its arguments. Projecting (9) on two orthogonal vectors $\partial_{m} a$ and $\epsilon_{m n} \partial^{n} a$, we get

$$
\begin{gathered}
\frac{1}{2} \partial_{m} a \mathcal{F}^{m}(\phi)=\partial_{m} a \partial^{m} \zeta, \\
\epsilon^{m n} \partial_{m} a \partial_{n} \zeta=0 .
\end{gathered}
$$

The general solution to (11) can be expressed in terms of an arbitrary function of $a(x)$

$$
\zeta=\hat{f}(a(x)) .
$$

We now notice that the left hand side of (10) transforms under (7) as follows

$$
\partial_{m} a \delta \mathcal{F}^{m}(\phi)=\partial_{m} a \partial^{m} f(a),
$$

Hence, we can use the transformations (7) to put $\zeta=0$. Then (10) reduces to the anti-self-duality (chirality) condition

$$
\partial_{m} \mathcal{F}^{m}=\epsilon^{m n} \partial_{m} \mathcal{F}_{n}=0 \quad \Rightarrow \quad \mathcal{F}_{m}(\phi)=\partial_{m} \phi-\epsilon_{m n} \partial^{n} \phi=0 .
$$




\subsection{Dualization of the chiral scalar}

To study duality properties of the action (5) with respect to a dualization of the field $\phi(x)$, we use a standard procedure. We replace (5) with the following classically equivalent action

$$
S=\int d^{2} x\left[-\frac{1}{2} F_{m} F^{m}+\frac{1}{2\left(\partial_{r} a\right)\left(\partial^{r} a\right)}\left(\partial^{m} a \mathcal{F}_{m}\right)^{2}+G^{m}\left(F_{m}-\partial_{m} \phi\right)\right] .
$$

In addition to the fields $a(x)$ and $\phi(x)$ this action now contains two independent auxiliary vector fields $G^{m}(x)$ and $F^{m}(x)$, and $\mathcal{F}_{m} \equiv F_{m}-\epsilon_{m n} F^{n}$. The variation of (14) with respect to $G^{m}$ gives the expression for the field $F_{m}$ in terms of $\phi$

$$
F_{m}=\partial_{m} \phi
$$

which, when substituted back into (14), yields the action (5). If we vary (14) with respect to $F_{m}$ we obtain the expression of $G^{m}$ in terms of $F^{m}$

$$
G_{m}=-\frac{2}{(\partial a)^{2}} \epsilon_{m n} \partial^{n} a\left(\partial_{p} a \mathcal{F}^{p}\right)+\epsilon_{m n} F^{n} .
$$

This can be inverted to express $F^{m}$ in terms of $G^{m}$

$$
\begin{gathered}
F_{m}=\frac{2}{(\partial a)^{2}} \partial_{m} a\left(\partial_{p} a \mathcal{G}^{p}\right)+\epsilon_{m n} G^{n}, \\
\mathcal{G}^{m} \equiv G^{m}-\epsilon^{m n} G_{n} .
\end{gathered}
$$

Note that on the mass shell (13) $G_{m}$ and $F_{m}$ are dual to each other in a usual sense, i.e. $G_{m}=\epsilon_{m n} F^{n}$.

Substituting (17) into the action (14) we get

$$
S=\int d^{2} x\left[\frac{1}{2} G_{m} G^{m}+\frac{1}{2\left(\partial_{r} a\right)\left(\partial^{r} a\right)}\left(\partial^{m} a \mathcal{G}_{m}\right)^{2}+\phi\left(\partial_{m} G^{m}\right)\right] .
$$

The variation of (18) with respect to $\phi$ gives

$$
\partial_{m} G^{m}=0 \Rightarrow G^{m}=\epsilon^{m n} \partial_{n} \psi \equiv \epsilon^{m n} F_{n}(\psi) .
$$

When the chirality condition (13) is satisfied, the expressions (16) and (15) take the form

$$
G_{m}=\epsilon_{m n} F^{n}=\epsilon_{m n} \partial^{n} \phi(x),
$$

from which it follows that on the mass shell the scalar $\psi(x)$ of (19) coincides with $\phi(x)$ up to a constant. Substituting (19) into (18) we again recover the action (5)).

Thus, as one might expect, the action (5) is self-dual with respect to a dualization of the chiral boson field $\phi$.

\subsection{Dualization of the auxiliary scalar}

Consider now properties of the action (5) under the dualization of the auxiliary scalar field $a(x)$. In order to do this we replace the action (5) with a classically equivalent action

$$
S=\int d^{2} x\left[-\frac{1}{2} F_{m}(\phi) F^{m}(\phi)+\frac{1}{2 u^{r} u_{r}}\left(u^{m} \mathcal{F}_{m}(\phi)\right)^{2}+v^{m}\left(u_{m}-\partial_{m} a\right)\right],
$$


where $F_{m}(\phi)=\partial_{m} \phi$, but $v^{m}(x)$ and $u^{m}(x)$ are now independent auxiliary fields. The equation of motion of the Lagrange multiplier $v^{m}$ gives

$$
u_{m}=\partial_{m} a
$$

while varying this action with respect to $u^{m}$, we obtain the following expression for the field $v^{m}$

$$
v^{m}=\frac{1}{\left(u^{r} u_{r}\right)^{2}} \epsilon^{m n} u_{n}\left(u^{p} \mathcal{F}_{p}(\phi)\right)^{2} .
$$

Its consequences are

$$
\begin{gathered}
v^{m} u_{m}=0, \quad v^{m} v_{m}=-\frac{1}{\left(u^{m} u_{m}\right)^{4}}\left(u^{p} \mathcal{F}_{p}(\phi)\right)^{4}, \\
\frac{u^{m}}{\sqrt{(u)^{2}}}=\epsilon^{m n} \frac{v_{n}}{\sqrt{-(v)^{2}}} .
\end{gathered}
$$

We see that normalized vectors $u_{m}$ and $v_{m}$ are dual to each other.

Substituting (23) into the action (20) we get

$$
S=\int d^{2} x\left[-\frac{1}{2} F_{m}(\phi) F^{m}(\phi)-\frac{1}{2 v^{r} v_{r}}\left(v^{m} \mathcal{F}_{m}(\phi)\right)^{2}+a\left(\partial_{m} v^{m}\right)\right] .
$$

Note that the second term of (24) has the opposite sign with respect to the analogous term in (20). The same difference in the sign we have observed when compared the Zwanziger action (2) with the action (3) for duality-symmetric Maxwell theory.

The equation of motion of the auxiliary field $a(x)$

$$
\partial_{m} v^{m}=0
$$

which follows from (24), allows us to express $v_{m}$ as a derivative of a scalar field

$$
v^{m}=\epsilon^{m n} \partial_{n} b(x) .
$$

Inserting this expression into (24) and taking into account the self-duality properties of $\mathcal{F}_{m}(\phi)$ we get the action

$$
S=\int d^{2} x\left[-\frac{1}{2} F_{m}(\phi) F^{m}(\phi)+\frac{1}{2\left(\partial_{r} b\right)\left(\partial^{r} b\right)}\left(\partial^{m} b \mathcal{F}_{m}(\phi)\right)^{2}\right]
$$

and find that it coincides with the action (5) up to the replacement of $a(x)$ with $b(x)$. Note that on the constraint surface (21), (23) and (25) the scalar fields are related through the following condition

$$
\frac{\partial_{m} a}{\sqrt{(\partial a)^{2}}}=\frac{\partial_{m} b}{\sqrt{(\partial b)^{2}}}
$$

We conclude that the action of two-dimensional chiral bosons is also self-dual under the dualization of the scalar field $a(x)$. 


\section{Dual descriptions of duality-symmetric Maxwell theory}

In this Section we study duality properties of a covariant duality-symmetric action for a free Maxwell field [10]. This action is the covariant generalization of the action (3) and has the form

$$
S=\int d^{4} x\left[-\frac{1}{8} F_{m n}^{\alpha}(A) F^{\alpha m n}(A)-\frac{1}{4\left(\partial^{s} a\right)\left(\partial_{s} a\right)} \partial^{m} a \mathcal{F}_{m n}^{\alpha}(A) \mathcal{F}^{\alpha n p}(A) \partial_{p} a\right]
$$

The action (27) is invariant under standard gauge transformations of $A_{m}^{\alpha}$ $\left(\delta A_{m}^{\alpha}=\partial_{m} \phi^{\alpha}(x)\right)$, and unusual local transformations

$$
\begin{gathered}
\delta A_{m}^{\alpha}=\left(\partial_{m} a\right) \Phi^{\alpha}, \\
\delta a=\phi, \quad \delta A_{m}^{\alpha}=\frac{\phi}{(\partial a)^{2}} \epsilon^{\alpha \beta} \mathcal{F}_{m n}^{\beta}(A) \partial^{n} a,
\end{gathered}
$$

(where $\Phi^{\alpha}(x)$ and $\phi(x)$ are corresponding parameters). The first symmetry (eq. (28)) is the covariant analogue of the symmetry (4) of the action (3) [7]. It reduces the $A^{\alpha}(x)$-field equations of motion to the duality relation between the field strengths $F_{m n}^{\alpha}(A)$

$$
\mathcal{F}_{m n}^{\alpha}(A)=\epsilon^{\alpha \beta} F_{m n}^{\beta}(A)-\frac{1}{2} \epsilon_{m n p q} F^{\alpha p q}(A)=0,
$$

and the second symmetry (29) allows one to gauge fix $\partial_{m} a(x)$ to be a constant time-like or space-like vector, upon which the action reduces to (3) (see [10] for details).

As in the $D=2$ case, let us perform a duality transformation of the action (27) by dualizing the vector $u_{m}=\partial_{m} a(x)$. For this replace the action (27) with an equivalent action 2

$$
S=\int d^{4} x\left(-\frac{1}{8} F_{m n}^{\alpha}(A) F^{\alpha m n}(A)-\frac{1}{4 u^{s} u_{s}} u^{m} \mathcal{F}_{m n}^{\alpha}(A) \mathcal{F}^{\alpha n p}(A) u_{p}+v^{m}\left(u_{m}-\partial_{m} a\right)\right),
$$

where $u^{m}(x)$ is now an independent auxiliary vector field. Variation of the action (31) with respect to the Lagrange multiplier $v^{m}$ gives the expression

$$
u_{m}=\partial_{m} a
$$

which, after substitution into (31), reduces it to the action (27).

The variation of the action with respect to $a(x)$ yields the relation

$$
\partial_{m} v^{m}=0 \quad \Rightarrow \quad v^{m}=\epsilon^{m n p q} \partial_{n} B_{p q}
$$

We observe that, as in the $D=2$ case, the vectors $u_{m}$ and $v_{m}$ have a dual realization. The first one is a vector "field strength" of the scalar field $a(x)$, while the second one is the dual field strength of a two-form field $B_{m n}(x)$.

Finally, if we vary this action with respect to the field $u^{m}$, we obtain the following equivalent expressions for $v^{m}$

$$
v^{m}=-\frac{1}{2\left(u^{s} u_{s}\right)^{2}} u^{m} u^{n} \mathcal{F}_{n p}^{\alpha}(A) \mathcal{F}^{\alpha p q}(A) u_{q}+\frac{1}{2\left(u^{s} u_{s}\right)} \mathcal{F}^{\alpha m n}(A) \mathcal{F}_{n p}^{\alpha}(A) u^{p}
$$

\footnotetext{
${ }^{2} \mathrm{An}$ action of this kind was discussed in 10 .
} 


$$
v^{m}=\frac{1}{2\left(u^{s} u_{s}\right)^{2}} \epsilon^{\alpha \beta} \epsilon^{m n p q} u_{n} \mathcal{F}_{p}^{\alpha} \mathcal{F}_{q}^{\beta},
$$

where in (35) we have denoted with $\mathcal{F}_{m n}^{\alpha}(A) u^{n} \equiv \mathcal{F}_{m}^{\alpha}$ two vectors orthogonal to $u^{m}$.

Eq. (35) is a kind of a duality relation between $v^{m}$ and $u_{m}$ which generalizes eq. (23) of the two-dimensional chiral-boson model of Section 2. It has been obtained from (34) by substituting $\mathcal{F}^{\alpha m n}$ in the second term of (34) with its identical expression

$$
\mathcal{F}^{\alpha m n}(A) \equiv-\frac{2}{\left(u^{s} u_{s}\right)} u^{[m} \mathcal{F}^{\alpha n]}-\frac{1}{\left(u^{s} u_{s}\right)} \epsilon^{\alpha \beta} \epsilon^{m n p q} u_{p} \mathcal{F}_{q}^{\beta} .
$$

From (34) and (35) it follows that

$$
v^{m} u_{m}=0, \quad v^{m} \mathcal{F}_{m n}^{\alpha}(A) u^{n} \equiv v^{m} \mathcal{F}_{m}^{\alpha}=0
$$

and

$$
\frac{1}{\left(v^{s} v_{s}\right)} v^{n} \mathcal{F}_{n p}^{\alpha}(A) \mathcal{F}^{\alpha p q}(A) v_{q}=-\frac{1}{\left(u^{s} u_{s}\right)} u^{n} \mathcal{F}_{n p}^{\alpha}(A) \mathcal{F}^{\alpha p q}(A) u_{q}
$$

The latter is obtained by contracting (36) with $v^{m}$, taking the square of both of its sides and using eqs. (37). Note that the four vectors $u^{n}, v^{m}$ and $\mathcal{F}^{\alpha m}$ form an orthogonal basis in $D=4$ space-time if $\mathcal{F}^{\alpha m}$ are nonzero. This holds off the mass shell (i.e. when eq. (30) is not satisfied), so that the duality transformation which we perform is an essentially offshell transformation, for which the mass-shell condition is a singular point. However, the action obtained after this duality transition again yields the duality relation (30) between the two gauge potentials, as we shall see below.

Substituting (38) into (31), we get the following action dual to (27)

$$
S=\int d^{4} x\left(-\frac{1}{8} F_{m n}^{\alpha}(A) F^{\alpha m n}(A)+\frac{1}{4 v^{s} v_{s}} v^{m} \mathcal{F}_{m n}^{\alpha}(A) \mathcal{F}^{\alpha n p}(A) v_{p}\right)
$$

Its second term has an opposite sign to that in (27) and contains the auxiliary field $v^{m}=\epsilon^{m n p q} \partial_{n} B_{p q}$ (see (33)), which can be gauge fixed to a constant vector by use of a local symmetry of (39). After this gauge fixing the action (39) reduces to the Zwanziger action (2). To convince oneself that such a symmetry does exist, perform a general variation of the action (39) with respect to the independent fields $A_{m}^{\alpha}$ and $B_{m n}$. This variation can be written in the following form

$$
\begin{gathered}
\delta S=\int d^{4} x\left[2\left(\delta A_{n}^{\alpha}-\frac{2}{v^{2}} \delta B_{n q} \mathcal{F}^{\alpha q r}(A) v_{r}\right) \epsilon^{\alpha \beta} \partial_{m}\left(\frac{1}{v^{2}} v^{[m} \mathcal{F}^{n] p \beta}(A) v_{p}\right)\right. \\
\left.+\frac{2}{v^{2}} \delta B_{m n} v^{m} \mathcal{F}^{\alpha l p}(A) v_{p} \epsilon^{\alpha \beta} \partial_{l}\left(\frac{1}{v^{2}} \mathcal{F}^{\beta n q}(A) v_{q}\right)\right] .
\end{gathered}
$$

The variation (40) vanishes when

$$
\begin{gathered}
\delta B_{m n}=-\frac{1}{2} \epsilon_{m n p q} v^{p} \Lambda^{q} \Rightarrow \delta v^{m}=2 \partial_{n}\left(v^{[m} \Lambda^{n]}\right)=\Lambda^{n} \partial_{n} v^{m}-v^{n} \partial_{n} \Lambda^{m}-v^{m} \partial_{n} \Lambda^{n} \\
\delta A^{m \alpha}=-\frac{1}{v^{s} v_{s}} \epsilon^{m n p q} v_{n} \Lambda_{p} \mathcal{F}_{q t}^{\alpha}(A) v^{t}
\end{gathered}
$$

and

$$
\delta B_{m n}=2 \partial_{[m} C_{n]},
$$


where $\Lambda^{m}(x)$ and $C^{m}(x)$ are vector parameters of these transformations. When $\Lambda_{m}$ in (41) is proportional to $v_{m}$, the variation of $B_{m n}$ becomes trivial. Therefore, only three independent components of $\Lambda^{m}(x)$ participate in (41).

The form of the transformation of $v^{m}$ in (41) is reminiscent of general coordinate transformations of vectors in curved space-time and differs from the latter only by the presence of the last term, which looks like a dilatation. Thus, by use of (41) and (42) we can gauge the normalized vector $\frac{v^{m}}{\sqrt{v^{r} v_{r}}}$ to a unit constant space-like vector $n^{m}$ ?. After such a gauge fixing the off-shell model loses the standard manifest Lorentz invariance. The latter acquires a modified form of a combination of the standard Lorentz transformations with the transformation (41) whose parameter is chosen in such a way that these combined transformations do not affect the constant vector $n^{m}$.

Standard Lorentz transformations of the 4 -vector $n^{m}$ are

$$
\delta_{L} n^{m}=\Omega^{m}{ }_{n} n^{n}
$$

where $\Omega_{m n}$ is an antisymmetric infinitesimal Lorentz parameter. At the same time we transform the vector $n^{m}$ as in (41):

$$
\delta_{\Lambda} n^{m}=\left(n^{q} \partial_{q}\right)\left(n^{m} n^{p} \Lambda_{p}-\Lambda^{m}\right)
$$

(on the r.h.s. of this relation the gauge fixing condition $\frac{v^{m}}{\sqrt{v^{r} v_{r}}}=n^{m}$ has been taken into account). Using this transformation we can compensate the Lorentz rotation (43) of $n^{m}$ if the parameter $\Lambda_{m}$ is chosen to be $\Lambda_{m}=\Omega_{m n} x^{n}$. Then

$$
\left(\delta_{L}+\delta_{\Lambda}\right) n^{m}=0
$$

But this combined transformation acts non-trivially on the fields $A_{m}^{\alpha}$. As a result the modified Lorentz transformation of $A_{m}^{\alpha}$ takes the following form

$$
\delta A_{m}^{\alpha}=\Omega_{m}{ }^{n} A_{n}^{\alpha}+\Omega^{p q}\left(x_{p} \partial_{q}\right) A_{m}^{\alpha}-\epsilon_{m n p q} n^{n} \Omega^{p r} x_{r} \mathcal{F}^{\alpha q t}(A) n_{t},
$$

The transformations (45) reduce to the standard Lorentz transformations on the mass shell, when their last term (proportional to $\mathcal{F}_{m n}^{\alpha}(A)$ ) vanishes. This term appeared due to the contribution of the transformations (41) with the parameter $\Lambda^{m}=\Omega^{m n} x_{n}$.

Substituting the gauge condition $\frac{v^{m}}{\sqrt{v^{r} v_{r}}}=n^{m}$ back into the covariant action (39) we reduce it to the free Zwanziger action (2). Thus the Zwanziger action possesses a non-manifest space-time symmetry (45), which one may regard as a dual analogue of the corresponding space-time symmetry of the non-covariant duality symmetric actions proposed in [5, 7].

From the general variation (40) of the action we derive the equations of motion of $A_{m}^{\alpha}$ and $B_{m n}$

$$
\begin{gathered}
\epsilon^{\alpha \beta} \partial_{m}\left(\frac{1}{v^{2}} v^{[m} \mathcal{F}^{n] p \beta}(A) v_{p}\right)=0, \\
\epsilon^{\alpha \beta} v^{[n} \partial_{l}\left(\frac{1}{v^{2}} \mathcal{F}^{m] r \beta} v_{r}\right) \mathcal{F}^{\alpha l p}(A) v_{p}=0 .
\end{gathered}
$$

\footnotetext{
${ }^{3}$ Notice that $B_{m n}$ enters the action (39) through the normalized vector field strength $\frac{v^{m}}{\sqrt{v^{r} v_{r}}}$ which has three independent components.
} 
The second-order differential equation (46) can be reduced to the first-order duality condition (30) th by choosing appropriate boundary conditions for the gauge field strengths, as in [1], or equivalently by using the following semi-local symmetry of the action (39)

$$
\delta A_{m}^{\alpha}=\Phi_{m}^{\alpha}(x)-\frac{1}{v^{2}} v_{m}\left(v^{n} \Phi_{n}^{\alpha}\right) .
$$

The parameters $\Phi_{m}^{\alpha}$ in (48) are restricted to satisfy

$$
\left(v^{p} \partial_{p}\right)\left(\Phi_{m}^{\alpha}(x)-\frac{1}{v^{2}} v_{m}\left(v^{n} \Phi_{n}^{\alpha}\right)\right)+\left(\partial_{m} v^{p}\right)\left(\Phi_{p}^{\alpha}(x)-\frac{1}{v^{2}} v_{p}\left(v^{n} \Phi_{n}^{\alpha}\right)\right)=0 .
$$

We have not been able to solve eq. (49) in terms of unrestricted fields in a covariant way, but when the symmetry (41) is fixed by the gauge $\frac{v^{m}}{\sqrt{v^{2}}}=n^{m}$, a solution can be obtained. Then (48) and (49) reduce to

$$
\begin{gathered}
\delta A_{m}^{\alpha}=\Phi_{m}^{\alpha}(x)-n_{m}\left(n^{l} \Phi_{l}^{\alpha}\right), \\
n^{l} \partial_{l} \Phi_{m}^{\alpha}(x)=0 .
\end{gathered}
$$

The latter is easily solved in terms of functions of three independent arguments

$$
\Phi_{m}^{\alpha}=\Phi_{m}^{\alpha}\left(y^{l}\right), \quad y^{l}=x^{l}-n^{l}\left(n_{p} x^{p}\right), \quad y^{l} n_{l} \equiv 0 .
$$

This symmetry of the free Zwanziger action (2) is an analogue of the symmetry (7) of the $D=2$ chiral boson model in a non-covariant gauge when, for example, $a(x)=n^{m} \epsilon_{m n} x^{n}$. Note that the coordinates $x^{l}-n^{l}\left(n_{p} x^{p}\right)$ coincide with gauge fixed components of the vector $Y^{l}=\frac{1}{\sqrt{v^{2}}} \epsilon^{l m n p} v_{m} B_{n p}$, when $\frac{v^{m}}{\sqrt{v^{2}}}=n^{m}$ and $B_{m n}=\epsilon_{m n p q} n^{p} x^{q}$.

The transformations (48) - (52) can be used to reduce eq. (46) to the duality condition (30). The procedure is analogous to the one considered in the previous Section to get the $D=2$ chiral boson equation.

Note that upon gauge fixing $v^{m}$ eqs. (46) can be rewritten as Maxwell equations for a single field strength $F_{m n}$. (This observation was a starting point of Zwanziger for the construction of the action [1]):

$$
\partial_{m} F^{m n}=0, \quad \partial_{m} F^{* m n}=0,
$$

where

$$
\begin{gathered}
F_{m n} \equiv-2 n^{[m} F^{1 n] p} n_{p}+\epsilon^{m n p q} n_{p} F_{q r}^{2} n^{r}, \\
F^{* m n}=\frac{1}{2} \epsilon^{m n p q} F_{p q}=-2\left(n^{[m} F^{2 n] p} n_{p}\right)-\epsilon^{m n p q} n_{p} F_{q r}^{1} n^{r} .
\end{gathered}
$$

When the boundary conditions are chosen such that $F_{m n}^{1}=-F_{m n}^{2 *}$ (as in eq. (30)), the field strength $F_{m n}$ coincides with $F_{m n}^{1}$.

We have seen that the parameters of the transformations (48)-(52) depend only on three of the four space-time coordinates. Therefore, they do not reduce the number of

\footnotetext{
${ }^{4}$ Note that the $B_{m n}(x)$-field equation (47) is automatically satisfied when the duality condition holds, but if we do not reduce $A_{m}(x)$-field equations to the duality condition then (47) may impose additional restrictions on the form of $\mathcal{F}_{m n}^{\alpha}(A)$, which would be of interest to analyze. This situation is novel in comparison with equations of motion of $A_{m}^{\alpha}(x)$ and $a(x)$ obtained from the action (27). In that case the $a(x)$-field equation was a direct consequence of the $A_{m}^{\alpha}(x)$-field equations.
} 
four-dimensional physical degrees of freedom, and their fixing is equivalent to choosing appropriate boundary conditions on the fields of the model on the 3-dimensional hypersurface, as it was done in [1]. This is in contrast to the duality-symmetric version of Maxwell theory based on the action (27). The transformations (28) form a full-fledged local symmetry of (27), they yield first-class constraints [12] and eliminate degrees of freedom of $A_{m}^{\alpha}(x)$ reducing them to that of a single Maxwell field [7, 10]. This example teaches us that dual formulations of one and the same model may have very different symmetry structure.

One can also show that, as the $D=2$ action (5), the actions (27) and (39) are self-dual with respect to a dualization of the gauge field strengths. The proof is presented in the Appendix. This self-duality is quite natural and reflects a basic property of the chiral boson actions in any even space-time dimension [5, 7].

\section{Conclusion and discussion}

By the use of examples of a $D=2$ chiral scalar and $D=4$ Maxwell theory we have studied duality properties of a Lorentz-covariant action for chiral bosons [10].

The action was shown to be self-dual with respect to a dualization of the field strengths of the chiral fields.

The dualization of the auxiliary field (whose role in the action is to preserve Lorentz invariance) resulted in another form of the covariant duality-symmetric action, where the role of the auxiliary field is taken by a $(D-2)$-rank tensor field. This action has symmetry properties which are different from that of its dual partner. Even though only the $D=2$ and $D=4$ case have been considered in detail, we have checked that dual actions for chiral bosons of this kind exist in any even space-time dimension.

In the $D=4$ case it has been shown that the dual covariant action (39) for a Maxwell field possesses a local symmetry which allows one to gauge fix the vector field strength of the auxiliary field $B_{m n}(x)$ to be a constant vector, and this reduces the action to the non-covariant Zwanziger action. As a consequence, we have established the duality relationship between the two duality-symmetric actions for free Maxwell theory.

We have also found a hidden space-time invariance of the Zwanziger action which on the mass shell becomes a conventional Lorentz invariance.

A problem which requires further study is the relationship between the two actions when they are coupled to dyonic sources.

Consistent coupling of the vector fields $A_{m}^{\alpha}(x)$ in the action (3) (and its covariant generalization (27)) to charged currents $j_{m}^{\alpha}(x)$ requires incorporation of non-local terms which can be interpreted as a contribution of a Dirac string attached to magnetically charged objects [15, 16, 17]. In the formulations under consideration the introduction of such terms is a requirement of local symmetries (28) and (29) which must be preserved when the coupling is made [17].

Consider this in more detail. To generalize the action (27) to one which describes a coupling of $A_{m}^{\alpha}(x)$ to currents $j_{m}^{\alpha}(x)$ we replace the field strengths of the gauge fields in (27) with modified stress tensors

$$
F^{\alpha m n}(A) \quad \rightarrow \quad \hat{F}^{\alpha m n}(A)=F^{\alpha m n}(A)+\frac{1}{2} \epsilon^{\alpha \beta} \epsilon^{m n p q} S_{p q}^{\beta}
$$


containing a tensor field $S_{m n}^{\alpha}$ determined by the condition

$$
\partial^{m} S_{m n}^{\alpha}+j_{n}^{\alpha}=0
$$

and add to the action the minimal interaction term. As a result we obtain the following action

$$
S=\int d^{4} x\left(-\frac{1}{8} \hat{F}_{m n}^{\alpha} \hat{F}^{\alpha m n}-\frac{1}{4 u^{s} u_{s}} u^{m} \hat{\mathcal{F}}_{m n}^{\alpha} \hat{\mathcal{F}}^{\alpha n p} u_{p}-\frac{1}{2} j^{m \alpha} A_{m}^{\alpha}\right)
$$

$\left(\hat{\mathcal{F}}^{\alpha m n} \equiv \epsilon^{\alpha \beta} \hat{F}^{\beta m n}-\frac{1}{2} \epsilon^{m n p q} \hat{F}_{p q}^{\alpha}\right)$. One may check that this action possesses the symmetries (28) and (29) if we replace all $F_{m n}^{\alpha}(A)$ with $\hat{F}_{m n}^{\alpha}(A)$ in the transformation laws. Using these symmetries we can obtain the modified duality condition

$$
\hat{\mathcal{F}}_{m n}^{\alpha}=0 \quad \Rightarrow \quad \hat{F}_{m n}^{1}=-\frac{1}{2} \epsilon_{m n p q} \hat{F}^{2 p q}
$$

as a solution to the equations of motion of this model. Differentiating (56) and taking into account (54) we get the Maxwell equations with electric and magnetic currents

$$
\partial_{m} \hat{F}^{1 m n}=j^{1 n}, \quad \partial_{m}^{*} \hat{F}^{1 m n}=j^{2 n} .
$$

Dualizing the action (55) we arrive at a generalization of the action (39), which has the same form as (55) but with $v^{m}(x)$ instead of $u^{m}(x)$ and with the plus sign in front of the second term. This Lorentz covariant action is invariant under transformations (41) and (48) with modified field strengths (53), and yields eqs. (56) and (57) as a consequence of equations of motion and gauge fixing. When the auxiliary vector is fixed to be a constant the action reduces to a non-covariant action, which differs in the non-local coupling terms from the original Zwanziger action [1] which also describes an electromagnetic interaction of dyons. The latter contains, in addition to the action (2), only the minimal coupling term $\int d^{4} x A_{m}^{\alpha}(x) j^{\alpha m}$. This Zwanziger action is not invariant under the transformations (45) and (50), and eqs. (56), (57) are singled out as particular solutions of $A_{m}^{\alpha}$-field equations by choosing appropriate boundary conditions [1].

We have now seen that for the classical duality-symmetric action of chiral bosons coupled to charged sources to be manifestly or non-manifestly space-time invariant the electric-magnetic coupling should be non-local. In spite of this fact, one may expect that the quantization of these actions will give the same result as Zwanziger quantization [1], since the space of quantized physical modes is one and the same in both cases.

Acknowledgements D.S. is grateful to Paolo Pasti and Mario Tonin for useful discussions. The work of A.M. and D.S. was partially supported by the INTAS Grants N 93-493-ext and N 97-0308. D.S. also thanks the Alexander von Humboldt Foundation for providing him with a European Research Grant for visiting Padua University where this work was completed.

\section{Appendix. Self-duality of chiral boson actions}

Consider the following action, equivalent to (27)

$$
S=\int d^{4} x\left(-\frac{1}{8} F_{m n}^{\alpha} F^{\alpha m n}-\frac{1}{4\left(\partial^{s} a\right)\left(\partial_{s} a\right)} \partial^{m} a \mathcal{F}_{m n}^{\alpha} \mathcal{F}^{\alpha n p} \partial_{p} a+\frac{1}{4} G_{m n}^{\alpha}\left(F^{\alpha m n}-2 \partial^{[m} A^{n] \alpha}\right)\right),
$$

\footnotetext{
${ }^{5}$ Here and below the vector fields $v^{m}$ and $u^{m}$ are defined as in the previous Section (see (32) and (33)).
} 
containing now antisymmetric tensor fields $F_{m n}^{\alpha}$ and $G_{m n}^{\alpha}$, two vector fields $A_{m}^{\alpha}$ and one scalar field $a(x)$ as independent ones.

Variation of the action (58) with respect to the Lagrange multiplier $G_{m n}^{\alpha}$ yields the expression for $F_{m n}^{\alpha}$ as the field strengths of the vector fields $A_{m}^{\alpha}$. If we substitute this relation back into the action (58), the latter reduces to (27).

To get the action dual to (27) with respect to $F_{m n}^{\alpha}$ and $G_{m n}^{\alpha}$ consider the $F_{m n}^{\alpha}$-variation of (58). This variation yields a duality-like relation

$$
G^{\alpha m n}=-\frac{2}{(\partial a)^{2}} \epsilon^{m n p q} \partial_{p} a \mathcal{F}_{q r}^{\alpha} \partial^{r} a-\frac{1}{2} \epsilon^{\alpha \beta} \epsilon^{m n p q} F_{p q}^{\beta},
$$

which expresses the fields $G_{m n}^{\alpha}$ through $F_{m n}^{\alpha}$ and becomes the standard duality relation on the mass shell (30). By performing algebraic manipulations we can invert this relation such that it will express $F_{m n}^{\alpha}$ in terms of $G_{m n}^{\alpha}$

$$
F_{m n}^{a}=\frac{4}{(\partial a)^{2}} \epsilon^{\alpha \beta} \partial^{[m} a \mathcal{G}^{n] p \beta} \partial_{p} a-\frac{1}{2} \epsilon^{\alpha \beta} \epsilon^{m n p q} G_{p q}^{\beta},
$$

where $\mathcal{G}_{m n}^{\alpha}=\epsilon^{\alpha \beta} G_{m n}^{\beta}-\frac{1}{2} \epsilon^{m n p q} G_{p q}^{\alpha}$.

If we vary the action (58) with respect to $A_{m}^{\alpha}$, we obtain the equations

$$
\partial_{m} G^{\alpha m n}=0
$$

which can be regarded as Bianchi identities for a dual field strength, whose general solution can be written in terms of two vector fields $B_{m}^{\alpha}$

$$
G^{\alpha m n}(B)=-\epsilon^{\alpha \beta} \epsilon^{m n p q} \partial_{p} B_{q}^{\beta} \equiv-\frac{1}{2} \epsilon^{\alpha \beta} \epsilon^{m n p q} F_{p q}^{\beta}(B)
$$

(Note that on the mass shell the $A_{m}^{\alpha}$ and $B_{m}^{\beta}$ fields coincide). Inserting this expression for $G_{m n}^{\alpha}$ into (60) and then plugging the latter into the action (58), we recover (27), where the gauge fields are $B_{m}^{\alpha}$

$$
S=\int d^{4} x\left[-\frac{1}{8} F_{m n}^{\alpha}(B) F^{\alpha m n}(B)-\frac{1}{4\left(\partial^{s} a\right)\left(\partial_{s} a\right)} \partial^{m} a \mathcal{F}_{m n}^{\alpha}(B) \mathcal{F}^{\alpha n p}(B) \partial_{p} a\right] .
$$

Thus, we conclude that, as one should expect, the action (27) is self-dual under the dualization of $A_{m}^{\alpha}$.

In the same way one can show that chiral boson actions in any dimension $D=2(p+1)$ are self-dual, which in fact is their basic property.

\section{References}

[1] D. Zwanziger, Phys. Rev. D3 (1971) 880.

[2] S. Deser and C. Teitelboim, Phys. Rev. D13 (1976) 1592;

S. Deser, J. Phys. A Math. Gen. 15 (1982) 1053.

[3] N. Marcus and J.H. Schwarz, Phys. Lett. 115B (1982) 111.

[4] R. Floreanini and R. Jackiw, Phys. Rev. Lett. 59 (1987) 1873. 
[5] M. Henneaux and C. Teitelboim, in Proc. Quantum Mechanics of Fundamental Systems 2, Santiago, 1987, p. 79; Phys. Lett. 206B (1988) 650.

[6] A. Tseytlin, Phys. Lett. 242B (1990) 163; Nucl. Phys. B350 (1991) 395.

[7] J.H. Schwarz and A. Sen, Nucl. Phys. B411 (1994) 35.

[8] W. Siegel, Nucl. Phys. B238 (1984) 307.

[9] B. McClain, Y. S. Wu and F. Yu, Nucl. Phys. B343 (1990) 689;

C. Wotzasek, Phys. Rev. Lett. 66 (1991) 129;

I. Martin and A. Restuccia, Phys. Lett. 323B (1994) 311;

F.P. Devecchi and M. Henneaux, Phys. Rev. D45 (1996) 1606;

I. Bengtsson and A. Kleppe, Int. J. Mod. Phys. A12 (1997) 3397;

N. Berkovits, Phys. Lett. 388B (1996) 743; Phys. Lett. 395B (1997) 28; Phys. Lett. 398B (1997) 79.

[10] P. Pasti, D. Sorokin and M. Tonin, Phys. Lett. 352B (1995) 59; Phys. Rev. D52 (1995) R4277.

[11] P.Pasti, D. Sorokin and M. Tonin, in Leuven Notes in Mathematical and Theoretical Physics, (Leuven University Press) Series B v.6, (1996) 167.

[12] P. Pasti, D. Sorokin and M. Tonin, Phys. Rev. D55 (1997) 6292.

[13] S. Cherkis and J. H. Schwarz, Phys. Lett. B403 (1997) 225.

[14] R. Amorim and J. Barcelos-Neto, Z. Phys. C68 (1995) 513.

[15] P.A.M. Dirac, Phys. Rev. 74 (1948) 817.

[16] S. Deser, A. Gomberoff, M. Henneaux and C. Teitelboim, Phys. Lett. 400B (1997) 80.

[17] R. Medina and N. Berkovits, Phys. Rev. D56 (1997) 6388. 